\title{
Convexity of Minimal Dominating and Total Dominating Functions of Corona Product Graph of a Cycle with a Complete Graph
}

\author{
M.Siva Parvathi \\ Dept. of Mathematics, K.R.K. Govt. Degree \\ College, Addanki-523201, Andhra Pradesh, India
}

\author{
B.Maheswari \\ Dept. of Applied Mathematics, S.P.Women's \\ University, Tirupat-517502, Andhra Pradesh, India
}

\begin{abstract}
'Domination in graphs' has been studied extensively and at present it is an emerging area of research in graph theory. An introduction and an extensive overview on domination in graphs and related topics is surveyed and detailed in the two books by Haynes et al. [1,2].

Product of graphs occurs naturally in discrete mathematics as tools in combinatorial constructions. They give rise to an important classes of graphs and deep structural problems. In this paper we study the dominating and total dominating functions of corona product graph of a cycle with a complete graph.
\end{abstract}

\section{Keywords}

Corona Product, Cycle, Complete Graph, Dominating function, Total dominating function.

Subject Classification: 68R10

\section{INTRODUCTION}

Domination Theory is an important branch of Graph Theory that has many applications in Engineering, Communication Networks, mobile computing, resource allocation, telecommunication and many others. Allan, R.B. and Laskar, R.[3], Cockayne, E.J. and Hedetniemi, S.T. [4] have studied various domination parameters of graphs.

Recently, dominating functions in domination theory have received much attention. The concepts of total dominating functions and minimal total dominating functions are introduced by Cockayne et al. [5]. Jeelani Begum, S. [6] has studied some total dominating functions of Quadratic Residue Cayley graphs.

Frucht and Harary [7] introduced a new product on two graphs $G_{1}$ and $G_{2}$, called corona product denoted by $G_{1} \odot G_{2}$. The object is to construct a new and simple operation on two graphs $G_{1}$ and $G_{2}$ called their corona, with the property that the group of the new graph is in general isomorphic with the wreath product of the groups of $\mathrm{G}_{1}$ and of $\mathrm{G}_{2}$.

The authors have studied some dominating functions of corona product graph of a cycle with a complete graph [8] and published papers on minimal dominating functions, some variations of $\mathrm{Y}$ - dominating functions and $\mathrm{Y}$ - total dominating functions $[9,10,11]$.

In this paper we discuss the convexity of minimal dominating and total dominating functions of corona product graph of a cycle with a complete graph

\section{CORONA PRODUCT OF $C_{n}$ AND $\boldsymbol{K}_{m}$}

The corona product of a cycle $C_{n}$ with a complete graph $K_{m}$ is a graph obtained by taking one copy of a $\mathrm{n}$ - vertex graph $C_{n}$ and $\mathrm{n}$ copies of $K_{m}$ and then joining the $i^{\text {th }}$ vertex of $C_{n}$ to every vertex of $i^{t h}$ copy of $K_{m}$ and it is denoted by $C_{n} \odot K_{m}$.

\section{CONVEXITY OF MINIMAL DOMINATING FUNCTIONS}

A study of convexity and minimality of dominating functions (MDFs) are given in Cockayne et al.[12] and $\mathrm{Yu}[13]$. Rejikumar [14] developed a necessary and sufficient condition for the convex combination of two MDFs to be again a MDF. Jeelani Begum [6] studied convexity of MDFs of Quadratic Residue Cayley Graphs.

In this section we discuss the convexity of minimal dominating functions of the corona product graph $G=$ $C_{n} \odot K_{m}$. First we define the convex combination of functions and prove some results on the convexity of MDFs of $G$.

Definition: Let $G(V, E)$ be a graph. Let $f$ and $g$ be two functions from $V$ to $[0,1]$ and $\lambda \in(0,1)$. Then the function $h: V \rightarrow[0,1]$ defined by $h(v)=\lambda f(v)+(1-\lambda) g(v)$ is called a convex combination of $f$ and $g$.

Definition: Let $G(V, E)$ be a graph. A subset $D$ of $V$ is said to be a dominating set (DS) of $G$ if every vertex in $V-D$ is adjacent to some vertex in $D$.

A dominating set $D$ is called a minimal dominating set (MDS) if no proper subset of $D$ is a dominating set of $\mathrm{G}$.

Definition: The domination number of $G$ is the minimum cardinality taken over all minimal dominating sets in $G$ and is denoted by $\chi(G)$.

Definition: Let $G(V, E)$ be a graph. A function $f: V \rightarrow[0,1]$ is called a dominating function (DF) of $G$ if $f(N[v])=$ $\sum_{u \in N[v]} f(u) \geq 1$, for each $v \in V$.

Definition: Let $f$ and $g$ be functions from $V$ to $[0,1]$. We define $f<g$ if $f(u) \leq g(u)$ for all $u \in V$, with strict inequality for at least one vertex $u \in V$. 
A dominating function $f$ of $G$ is called a minimal dominating function (MDF) if for all $g<f, g$ is not a dominating function.

Theorem 3.1: Let $D_{1}$ and $D_{2}$ be two MDSs of $G=C_{n} \odot K_{m}$ Let $f_{1}: V \rightarrow[0,1]$ and $f_{2}: V \rightarrow[0,1]$ be defined by

$$
f_{1}(v)= \begin{cases}1, & \text { if } v \in D_{1}, \\ 0, & \text { otherwise }\end{cases}
$$

and $f_{2}(v)= \begin{cases}1, & \text { if } \mathrm{v} \in D_{2}, \\ 0, & \text { otherwise }\end{cases}$

Then the convex combination of $f_{1}$ and $f_{2}$ becomes a MDF of $G=C_{n} \odot K_{m}$.

Proof: Let $D_{1}$ and $D_{2}$ be two MDSs of G. Let $f_{1}, f_{2}$ be two functions defined as in the hypothesis.

These functions become MDFs of $G=C_{n} \odot K_{m}$ [8].

Let $h(v)=\alpha f_{1}(v)+\beta f_{2}(v)$, where $\alpha+\beta=1$ and $0<\alpha<1,0<\beta<1$.

Case 1: Suppose $D_{1} \cap D_{2} \neq \varphi$.

For $v \in V$, the possible values of $h(v)$ are

$$
h(v)= \begin{cases}\alpha, & \text { if } \mathrm{v} \in D_{1}-D_{2}, \\ \beta, & \text { if } \mathrm{v} \in D_{2}-D_{1}, \\ \alpha+\beta, & \text { if } \mathrm{v} \in D_{1} \cap D_{2}, \\ 0, & \text { otherwise }\end{cases}
$$

Then

$\sum h(u)=s \alpha+t \beta$, if $\mathrm{s}$ - vertices of $\mathrm{D}_{1}$ and $\mathrm{t}$ - vertices of $\mathrm{D}_{2}$ are in $\mathrm{N}[\mathrm{v}]$

So $\sum_{u \in N[v]} h(u) \geq 1, \quad \forall v \in \mathrm{V}$.

This implies that $h$ is a DF.

Now we check for the minimality of $h$.

Define $g: V \rightarrow[0,1]$ by

$$
g(v)= \begin{cases}\mathrm{r}, & \text { if } \mathrm{v}=\mathrm{v}_{\mathrm{i}} \in D_{1} \cap D_{2} \\ \alpha+\beta, & \text { if } \mathrm{v} \in\left(\mathrm{D}_{1} \cap \mathrm{D}_{2}\right)-\left\{\mathrm{v}_{\mathrm{i}}\right\} \\ \alpha, & \text { if } \mathrm{v} \in \mathrm{D}_{1}-D_{2} \\ \beta, & \text { if } \mathrm{v} \in \mathrm{D}_{2}-D_{1} \\ 0, & \text { otherwise. }\end{cases}
$$

where $0<r<1$

Since strict inequality holds at the vertex $v_{i} \in V$, it follows that $g<h$.
Then $\sum_{u \in N[v]} g(u)$

$=$

$\mathrm{r}+\beta, \quad$ if $\mathrm{v} \in \mathrm{i}^{\text {th }}$ copy of $K_{m}$ in $\mathrm{G}$,

$\left\{s \alpha+t \beta+r\right.$, if $s$-vertices of $\mathrm{D}_{1}, \mathrm{t}$-vertices of $\mathrm{D}_{2}$ and $\mathrm{v}_{\mathrm{i}}$ are in $\mathrm{N}[\mathrm{v}]$

$s \alpha+t \beta, \quad$ if $s$-vertices of $\mathrm{D}_{1}$ and $\mathrm{t}$-vertices of $\mathrm{D}_{2}$ are in $\mathrm{N}[\mathrm{v}]$.

This implies that $\sum_{u \in N[v]} g(u)=r<1$, for the vertices in the

$i^{\text {th }}$ copy of $K_{m}$ in $G$.

So $g$ is not a DF.

Since $g$ is taken arbitrarily, it follows that there exists no $g<h$ such that $g$ is a DF.

Thus $h$ is a MDF.

Case 2: Suppose $D_{1} \cap D_{2}=\varphi$.

For $v \in V$, the possible values of $h(v)$ are

$h(v)= \begin{cases}\alpha, & \text { if } \mathrm{v} \in D_{1}, \\ \beta, & \text { if } \mathrm{v} \in D_{2}, \\ 0, & \text { otherwise }\end{cases}$

Then

$\sum_{u \in N[v]} h(u)=s \alpha+t \beta$, if $\mathrm{s}$ - vertices of $\mathrm{D}_{1}$ and $\mathrm{t}$ - vertices of $\mathrm{D}_{2}$ are in $\mathrm{N}[\mathrm{v}]$

So $\sum_{u \in N[v]} h(u) \geq 1, \quad \forall v \in \mathrm{V}$.

This implies that $h$ is a DF.

Now we check for the minimality of $h$.

Define $g: V \rightarrow[0,1]$ by

$g(v)= \begin{cases}\mathrm{r}, & \text { if } \mathrm{v}=\mathrm{v}_{\mathrm{i}} \in D_{1} \\ \alpha, & \text { if } \mathrm{v} \in \mathrm{D}_{1}-\left\{v_{i}\right\} \\ \beta, & \text { if } \mathrm{v} \in \mathrm{D}_{2} \\ 0, & \text { otherwise. }\end{cases}$

where $0<r<\alpha$

Since strict inequality holds at the vertex $v_{i} \in V$, it follows that $g<h$

Then $\sum_{u \in N[v]} g(u)$
$=$
$\left\{\begin{array}{l}\mathrm{r}+\beta, \quad \text { if } \mathrm{v} \in \mathrm{i}^{\text {th }} \text { copy of } K_{m} \text { in } \mathrm{G}, \\ s \alpha+t \beta+r, \text { if } \mathrm{s} \text { - vertices of } \mathrm{D}_{1}, \mathrm{t}-\text { vertices of } \mathrm{D}_{2} \text { and } \mathrm{v}_{\mathrm{i}} \text { are in } \mathrm{N}[\mathrm{v}], \\ s \alpha+t \beta, \quad \text { if } \mathrm{s} \text { - vertices of } \mathrm{D}_{1} \text { and } \mathrm{t}-\text { vertices of } \mathrm{D}_{2} \text { are in } \mathrm{N}[\mathrm{v}]\end{array}\right.$

This implies that $\sum_{u \in N[v]} g(u)=r+\beta<\alpha+\beta=1$, for the vertices in the $i^{\text {th }}$ copy of $K_{m}$ in $G$. 
So $g$ is not a DF.

Since $g$ is taken arbitrarily, it follows that there exists no $g<h$ such that $g$ is a DF.

Thus $h$ is a MDF.

\section{CONVEXITY OF MINIMAL TOTAL DOMINATING FUNCTIONS}

The concepts of total dominating functions (TDFs) and minimal total dominating functions (MTDFs) are introduced by Cockayne et al. [15]. A study of convexity and minimality of TDFs are given in Cockayne et al. [12, 15]. Cockayne et al.[12] obtained a necessary and sufficient condition for the convex combination of two minimal total dominating functions to be again a minimal total dominating function.

The authors have studied minimal total dominating functions of $G=C_{n} \odot K_{m}$ [16]. In this section we consider minimal total dominating functions of corona product graph $G=C_{n} \odot K_{m}$ and discuss their convexity .

Definition: Let $G(V, E)$ be a graph without isolated vertices. A subset $T$ of $V$ is called a total dominating set (TDS) if every vertex in $V$ is adjacent to at least one vertex in $T$.

If no proper subset of $T$ is a total dominating set, then $T$ is called a minimal total dominating set (MTDS) of $G$.

Definition: The minimum cardinality of a MTDS of $G$ is called a total domination number of $G$ and is denoted by $\gamma_{t}(G)$.

Definition: Let $G(V, E)$ be a graph. A function $f: V \rightarrow$ $[0,1]$ is called a total dominating function (TDF) of $G$ if $f(N(v))=\sum_{u \in N(v)} f(u) \geq 1$, for each $v \in V$.

Definition: Let $f$ and $g$ be functions from $V$ to $[0,1]$. We define $f<g$ if $f(u) \leq g(u)$ for all $u \in V$, with strict inequality for at least one vertex $u \in V$.

A TDF $f$ of $G$ is called a minimal total dominating function (MTDF) if for all $g<f, g$ is not a TDF.

Theorem 4.1: Let $T_{1}$ and $T_{2}$ be two MTDSs of $G=$ $C_{n} \odot K_{m}$. Let $f_{1}: V \rightarrow[0,1]$ and $f_{2}: V \rightarrow[0,1]$ be defined by

$$
f_{1}(v)= \begin{cases}1, & \text { if } \mathrm{v} \in T_{1} \\ 0, & \text { otherwise }\end{cases}
$$

and

$$
f_{2}(v)= \begin{cases}1, & \text { if } \mathrm{v} \in T_{2} \\ 0, & \text { otherwise }\end{cases}
$$

Then the convex combination of $f_{1}$ and $f_{2}$ becomes a MTDF of $G=C_{n} \odot K_{m}$.

Proof: Let $T_{1}$ and $T_{2}$ be two MTDSs of $G$. Let $f_{1}, f_{2}$ be two functions defined as in the hypothesis.

These functions become MTDFs of $G=C_{n} \odot K_{m}$ [8].

Let $h(v)=\alpha f_{1}(v)+\beta f_{2}(v), \quad$ where $\alpha+\beta=1$ and $0<\alpha<1,0<\beta<1$.
Case 1: Suppose $T_{1} \cap T_{2} \neq \varphi$.

Then for $v \in V$, the possible values of $h(v)$ are

$$
h(v)= \begin{cases}\alpha, & \text { if } \mathrm{v} \in T_{1}-T_{2}, \\ \beta, & \text { if } \mathrm{v} \in T_{2}-T_{1}, \\ \alpha+\beta, & \text { if } \mathrm{v} \in T_{1} \cap T_{2}, \\ 0, & \text { otherwise }\end{cases}
$$

Now

$$
\sum_{u \in N(v)} h(u)=s \alpha+t \beta, \quad \text { if } \mathrm{s} \text { - vertices of } \mathrm{T}_{1} \text { and }
$$

$\mathrm{t}$ - vertices of $\mathrm{T}_{2}$ are in $\mathrm{N}(\mathrm{v})$.

Therefore $\sum_{u \in N(v)} h(u) \geq 1, \quad \forall \mathrm{v} \in \mathrm{V}$.

This implies that $h$ is a TDF.

Now we check for the minimality of $h$.

Define $g: V \rightarrow[0,1]$ by

$$
g(v)= \begin{cases}\mathrm{r}, & \text { if } \mathrm{v}=\mathrm{v}_{\mathrm{i}} \in T_{1} \cap T_{2}, \\ \alpha+\beta, & \text { if } \mathrm{v} \in\left(\mathrm{T}_{1} \cap \mathrm{T}_{2}\right)-\left\{\mathrm{v}_{\mathrm{i}}\right\} \\ \alpha, & \text { if } \mathrm{v} \in \mathrm{T}_{1}-T_{2}, \\ \beta, & \text { if } \mathrm{v} \in \mathrm{T}_{2}-T_{1}, \\ 0, & \text { otherwise. }\end{cases}
$$

where $0<r<1$.

Since strict inequality holds at the vertex $v_{i} \in T_{1} \cap T_{2}$, it follows that $g<h$.

Then $\sum_{u \in N(v)} g(u)$

$$
= \begin{cases}\mathrm{r}, & \text { if } \mathrm{v} \in \mathrm{i}^{\text {th }} \quad \text { copy of } \mathrm{K}_{\mathrm{m}} \text { in } \mathrm{G}, \\ s \alpha+t \beta+r, & \text { if } \mathrm{s}-\text { vertices of } \mathrm{T}_{1}, \mathrm{t}-\text { vertices of } \mathrm{T}_{2} \text { and } \mathrm{v}_{\mathrm{i}} \text { are in } \mathrm{N}(\mathrm{v}), \\ s \alpha+t \beta, & \text { if } \mathrm{s}-\text { vertices of } \mathrm{T}_{1} \text { and } \mathrm{t}-\text { vertices of } \mathrm{T}_{2} \text { are in } \mathrm{N}(\mathrm{v}) .\end{cases}
$$

This implies that $\sum_{u \in N(v)} g(u)=r<1$, for the vertices in the $i^{\text {th }}$ of $K_{m}$ in $G$.

So $g$ is not a TDF.

Since $g$ is taken arbitrarily, it follows that there exists no $g<h$ such that $g$ is a TDF.

Thus $h$ is a MTDF.

Case 2: Suppose $T_{1} \cap T_{2}=\varphi$.

Then for $v \in V$, the possible values of $h(v)$ are 
$h(v)= \begin{cases}\alpha, & \text { if } \mathrm{v} \in T_{1}, \\ \beta, & \text { if } \mathrm{v} \in T_{2}, \\ 0, & \text { otherwise. }\end{cases}$

Now

$\sum_{u \in N(v)} h(u)=s \alpha+t \beta$, if $\mathrm{s}-$ vertices of $\mathrm{T}_{1}$ and

$\mathrm{t}$ - vertices of $\mathrm{T}_{2}$ are in $\mathrm{N}(\mathrm{v})$.

Therefore $\sum_{u \in N(v)} h(u) \geq 1, \quad \forall \mathrm{v} \in \mathrm{V}$.

This implies that $h$ is a TDF.

Now we check for the minimality of $h$.

Define $g: V \rightarrow[0,1]$ by

$$
g(v)= \begin{cases}\mathrm{r}, & \text { if } \mathrm{v}=\mathrm{v}_{\mathrm{i}} \in T_{1}, \\ \alpha, & \text { if } \mathrm{v} \in \mathrm{T}_{1}-\left\{v_{i}\right\} \\ \beta, & \text { if } \mathrm{v} \in \mathrm{T}_{2}, \\ 0, & \text { otherwise. }\end{cases}
$$

where $0<r<\alpha$.

Since strict inequality holds at the vertex $v_{i} \in T_{1}$, it follows that $g<h$.

Then $\sum_{u \in N(v)} g(u)$

$=\left\{\begin{array}{l}\mathrm{r}+\beta, \quad \text { if } \mathrm{v} \in \mathrm{i}^{\text {th }} \text { copy of } \mathrm{K}_{\mathrm{m}} \text { in } \mathrm{G}, \\ s \alpha+t \beta+r, \text { if } \mathrm{s}-\text { vertices of } \mathrm{T}_{1}, \mathrm{t}-\text { vertices of } \mathrm{T}_{2} \text { and } \mathrm{v}_{\mathrm{i}} \text { are in } \mathrm{N}(\mathrm{v}), \\ s \alpha+t \beta, \quad \text { if } \mathrm{s}-\text { vertices of } \mathrm{T}_{1} \text { and } \mathrm{t}-\text { vertices of } \mathrm{T}_{2} \text { are in } \mathrm{N}(\mathrm{v}) .\end{array}\right.$

This implies that $\sum_{u \in N(v)} g(u)=r+\beta<\alpha+\beta=1$, for the vertices in the $i^{\text {th }}$ of $K_{m}$ in $G$.

So $g$ is not a TDF.

Since $g$ is taken arbitrarily, it follows that there exists no $g<h$ such that $g$ is a TDF.

Thus $h$ is a MTDF.

\section{CONCLUSION}

It is interesting to study the convexity of minimal dominating and total dominating functions of corona product graph of a cycle with a complete graph. This work gives the scope for an extensive study of dominating functions in general of these graphs.

\section{REFERENCES}

[1] Haynes, T.W., Hedetniemi, S.T. and Slater, P.J. Domination in Graphs: Advanced Topics, Marcel Dekker, Inc., New York, (1998).
[2] Haynes, T.W., Hedetniemi, S.T. and Slater, P.J. Fundamentals of domination in graphs, Marcel Dekker, Inc., New York , (1998).

[3] Allan, R.B. and Laskar, R.C. On domination, independent domination numbers of a graph. Discrete Math., 23 (1978), 73 - 76.

[4] Cockayne, E.J. and Hedetniemi, S.T. - Towards a theory of domination in graphs. Networks, 7 (1977), $247-261$.

[5] Cockayne, C.J., Dawes, R.M. and Hedetniemi, S.T Total domination in graphs, Networks, 10 (1980), $211-219$.

[6] Jeelani Begum, S. - Some studies on dominating functions of Quadratic Residue Cayley Graphs, Ph. D. thesis, Sri Padmavathi Mahila Visvavidyalayam,Tirupati, Andhra Pradesh, India, (2011).

[7] Frucht, R. and Harary, F. - On the corona of Two Graphs, Aequationes Mathematicae, Volume 4, Issue 3 (1970), $322-325$.

[8] Siva Parvathi, $\mathbf{M}$ - Some studies on dominating functions of corona product graphs, Ph.D thesis, Sri Padmavati Mahila Visvavidyalayam, Tirupati, Andhra Pradesh, India, (2013).

[9] Siva Parvathi, M and Maheswari, B . - Minimal Dominating Functions of Corona Product Graph of a Cycle with a Complete Graph - International Journal of Computer Engineering \& Technology, Volume 4, Issue 4 (2013), $248-256$

[10] Siva Parvathi, M and Maheswari, B. - Some variations of Y-Dominating Functions of Corona Product Graph of a Cycle with a Complete Graph - International Journal of Computer Applications, Volume 81, Issue 1 (2013), $16-21$

[11] Siva Parvathi, M and Maheswari, B. - Some variations of Total Y-Dominating Functions of Corona Product Graph of a Cycle with a Complete Graph - Fire Journal Science and Technology (accepted).

[12] Cockayne, E.J., Mynhardt, C.M. and Yu, B. Tota dominating functions in trees: Minimality and Convexity, Journal of Graph Theory, 19(1995), 83-92.

[13] Yu, B., Convexity of minimal total dominating functions in graphs, J. Graph Theory, 24 (4) (1997), 313 - 321.

[14] Reji Kumar, K., Studies in Graph Theory - Dominating functions, Ph.D. thesis, Manonmaniam Sundaranar University, Tirunelveli, India, (2004).

[15] Cockayne, E.J. and Mynhardt, C.M. Convexity of extremal domination - related functions of graphs. In: Domination in Graphs - Advaned Topics, (Ed.T.W.Haynes, S.T. Hedetniemi, P.J. Slater), Marcel Dekker, New York, (1998), 109 - 131.

[16] Siva Parvathi, M and Maheswari, B. - Minimal total dominating functions of corona product graph of a cycle with a complete graph - International Journal of Applied Information Systems (communicated). 